\title{
Modified Uncertainty Measure of Rough Fuzzy Sets from the Perspective of Fuzzy Distance
}

\author{
Jie Yang $\left(\mathbb{D},{ }^{1,2}\right.$ Taihua Xu $\mathbb{D}^{2},{ }^{2,3}$ and Fan $\mathrm{Zhao}^{2}$ \\ ${ }^{1}$ School of Physics and Electronic Science, Zunyi Normal University, Zunyi 563002, China \\ ${ }^{2}$ Chongqing Key Laboratory of Computational Intelligence, Chongqing University of Posts and Telecommunications, \\ Chongqing 400065, China \\ ${ }^{3}$ School of Information Science and Technology, Southwest Jiaotong University, Chengdu 610031, China
}

Correspondence should be addressed to Taihua Xu; xth19890410@163.com

Received 9 April 2018; Accepted 19 July 2018; Published 6 August 2018

Academic Editor: Paolo Manfredi

Copyright (C) 2018 Jie Yang et al. This is an open access article distributed under the Creative Commons Attribution License, which permits unrestricted use, distribution, and reproduction in any medium, provided the original work is properly cited.

As an extension of Pawlak's rough sets, rough fuzzy sets are proposed to deal with fuzzy target concept. As we know, the uncertainty of Pawlak's rough sets is rooted in the objects contained in the boundary region, while the uncertainty of rough fuzzy sets comes from three regions (positive region, boundary region, and negative region). In addition, in the view of traditional uncertainty measures, the two rough approximation spaces with the same uncertainty are not necessarily equivalent, and they cannot be distinguished. In this paper, firstly, a fuzziness-based uncertainty measure is proposed. Meanwhile, the essence of the uncertainty for rough fuzzy sets and its three regions in a hierarchical granular structure is revealed. Then, from the perspective of fuzzy distance, we introduce a modified uncertainty measure based on the fuzziness-based uncertainty measure and present that our method not only is strictly monotonic with finer approximation spaces, but also can distinguish the two rough approximation spaces with the same uncertainty. Finally, a case study is introduced to demonstrate that the modified uncertainty measure is more suitable for evaluating the significance of attributes. These works are useful for further study on rough sets theory and promote the development of uncertain artificial intelligence.

\section{Introduction}

The rough sets introduced by Pawlak $[1,2]$ have been demonstrated as an effective tool to handle uncertain information by using the given information granulations [3-5]. An uncertain concept can be described by a pair of lower and upper approximation sets. And three disjoint regions are constructed in a rough approximation space. Generally speaking, the target concepts in Pawlak's rough sets are usually accurate and crisp. For a decision-making problem, there are only two states which are opposite and disjoint each other for a crisp concept. For example, in the decisionmaking problems of diagnosis analysis, there are only two states of Yes or No for a patient. That is, a patient is either diseased or not diseased. However, in many real decisionmaking applications, the states of the target concept may be uncertain and fuzzy in practice. To address this problem, the rough fuzzy sets (RFS) [6-8] are proposed to deal with the target concept which is usually fuzzy or uncertain. In rough fuzzy sets, the lower and upper approximation fuzzy sets are considered as two boundary fuzzy sets of the target concept.

Probabilistic rough fuzzy set model [9], based on the average membership degree, classifies an approximation space into positive region, boundary region, and negative region by setting a pair of threshold $(\alpha, \beta)(0 \leq \beta<\alpha \leq 1)$. Similar to the probabilistic rough sets, the rough fuzzy sets also have a good fault-tolerance. It is well known that the uncertainty of classical rough sets is only rooted in the boundary region, while the uncertainty of rough fuzzy sets not only comes from the boundary region, but also comes from the negative or positive regions, because the membership degrees of these objects in the negative or positive regions are not completely equal to 1 or 0 . From the viewpoint of hierarchical quotient space $[10,11]$, with the increase of information, not only the objects in the boundary region, but also the objects in the positive region and negative region will be reclassified. 
Therefore, for rough fuzzy sets, the uncertainty comes from three regions and is not monotonic with finer knowledge spaces.

As a new methodology for simulating human cognitive mechanism, granular computing $(\mathrm{GrC})$ is regarded as an umbrella covering the theories, methodologies, techniques, and tools in artificial intelligence [12-17]. From the viewpoint of $\mathrm{GrC}$, the certainty and uncertainty of knowledge can be transformed for each other at a certain granularity level [18]. As the main GrC models, fuzzy sets [19], rough sets [1], quotient space [10], and cloud model [20] realize the representation and transformation of uncertain knowledge from the different views. The uncertainty measure plays an important role in acquiring rules from information system and it is helpful in evaluating attribute weight in attribute reduction. Wierman [21] first introduced an axiomatic definition of knowledge granularity to measure the uncertainty in information system. Yao [22] presented a so-called expected granularity (EG) class that provides a family of uncertain measure. Besides, there are various uncertain measures, such as fuzziness [23, 24], rough entropy [25], inclusion degree [26], roughness [27], and knowledge distance [28, 29].

The uncertain measure of rough fuzzy sets model has drawn many researchers' attention, because it can reflect the quality of acquiring rules from information system. Guo and $\mathrm{Mi}$ [30] proposed a fuzziness measure of rough fuzzy sets based on the conditional entropy. By using new lower and upper approximation operators, Beg and Rashid [31] proposed a modified soft rough fuzzy set model, which provide better approximations of undefinable sets. Based on rough entropy and information entropy, Wang [32] introduced a method address the uncertainty measure of rough fuzzy sets. Combining the rough degree with the rough entropy, Qin [33] proposed a new rough entropy that is strictly monotonic with finer approximation space. Sun $[34,35]$ introduced an uncertainty measure for generalized rough fuzzy sets based on the Shannon entropy, which is effective and suitable for evaluating the roughness and accuracy of a generalized rough fuzzy sets. From the perspective of distance, $\mathrm{Hu}$ [36] studied the roughness measure of rough fuzzy sets and applied it to incomplete information systems with fuzzy decision. However, there are still several shortcomings in the current research on uncertainty measure of rough fuzzy sets as follows: (i) lack of theoretical analysis on the change rules of uncertainty of RFS in a hierarchical granular structure. For example, in many practical applications, such as medical diagnosis [37], decision-making [38], and feature selection [39], the analysis on the uncertainty of three regions with changing knowledge spaces is helpful in improving the decision quality. (ii) In the view of traditional uncertainty measures, two rough approximation spaces with the same uncertainty are not necessarily equivalent and the difference between them is difficult to reflect. Actually, in some cases, such as granularity selection, attribute reduction, and multigranularity construction, we usually need to discriminate the two rough approximation spaces. To solve the above problems, in this paper, from the perspective of fuzzy distance, we propose a modified uncertainty measure based on fuzziness.
The rest of this paper is organized as follows. Section 2 presents many preliminary concepts such as rough fuzzy sets, step fuzzy set, average membership degree, average fuzzy set, and probabilistic rough fuzzy sets, etc. In Section 3, a basic uncertainty measure based on fuzziness is proposed to investigate the changing rules of uncertainty of RFS in a hierarchical granular structure. In Section 4, in the view of fuzzy distance, a modified uncertainty measure is presented. In Section 5, an illustrative example is conducted to verify the effectiveness of the proposed method. In Section 6, conclusions are drawn.

\section{Preliminaries}

In order to facilitate the description of this paper, many basic concepts are reviewed briefly in this section. In this paper, we denote an information system by $S=(U, C \cup D, V, f)$, where $U$ is a nonempty finite domain, $C$ is the set of condition attributes, $D$ is the decision attribute, $V$ is the set of all attribute values, and $f: U \times C$ is an information function.

Definition 1 (rough sets $[1,2]$ ). Given an information system $S=(U, C \cup D, V, f), R \subseteq C$, and $X \subseteq U$, the lower and upper approximation sets of $X$ are defined as follows:

$$
\begin{aligned}
& \underline{R}(X)=\left\{x \in U \mid[x]_{R} \subseteq X\right\}, \\
& \bar{R}(X)=\left\{x \in U \mid[x]_{R} \cap X \neq \phi\right\} .
\end{aligned}
$$

where $[x]_{R}$ denotes the equivalence class induced by $U / R$, namely, $U / R=\left\{[x]_{R}\right\}=\left\{[x]_{1},[x]_{2}, \ldots,[x]_{m}\right\}$.

For simplicity, let $[x]_{R} \triangleq[x]$ in case of confusion. If $\bar{R}(X)=\underline{R}(X), X$ is a definable set; otherwise $X$ is a rough set. Universe $U$ is divided by positive region, boundary region, and negative region, then the three regions can be defined, respectively, as follows:

$$
\begin{aligned}
\operatorname{POS}_{R}(X) & =\underline{R}(X), \\
\operatorname{BND}_{R}(X) & =\bar{R}(X)-\underline{R}(X), \\
\operatorname{NEG}_{R}(X) & =U-\bar{R}(X) .
\end{aligned}
$$

In this paper, a partition space $U / R$ is also called a knowledge space or granularity space. If $R_{1}, R_{2} \subseteq C, U / R_{1}=$ $\left\{p_{1}, p_{2}, \ldots, p_{l}\right\}$ and $U / R_{2}=\left\{q_{1}, q_{2}, \ldots, q_{m}\right\}$ are two knowledge spaces. If $\forall_{q_{j} \in U / R_{2}}\left(\exists_{p_{i} \in U / R_{1}}\left(q_{j} \subseteq p_{i}\right)\right)$, then $U / R_{2}$ is finer than $U / R_{1}$, denoted by $U / R_{2} \preceq U / R_{1}$. If $\forall_{q_{j} \in U / R_{2}}\left(\exists_{p_{i} \in U / R_{1}}\left(q_{j} \subset\right.\right.$ $\left.p_{i}\right)$ ), then $U / R_{2}$ is strictly finer than $U / R_{1}$, denoted by $U / R_{2} \prec$ $U / R_{1}$.

Definition 2 (rough fuzzy sets [6-8]). Given an information system $S=(U, C \cup D, V, f), R \subseteq C$ and $X$ is a fuzzy set on $U$, then the lower and upper approximation sets of $X$ can be defined as a pair of fuzzy sets, $\underline{R}(X)$ and $\bar{R}(X)$. And their membership functions are defined as follows:

$$
\begin{array}{ll}
\mu_{\underline{R}}(x)=\inf \left\{\mu_{X}(y) \mid y \subseteq[x]_{R}\right\}, & \forall x \in U, \\
\mu_{\bar{R}}(x)=\sup \left\{\mu_{X}(y) \mid y \subseteq[x]_{R}\right\}, & \forall x \in U,
\end{array}
$$


where $[x]_{R}$ denotes the equivalence class induced by the equivalence relation $U / R$. If $\underline{R}(X)=\bar{R}(X)$, then $X$ is a definable fuzzy set; otherwise $\bar{X}$ is a rough fuzzy sets.

Definition 3 (step fuzzy set [40]). Given an information system $S=(U, C \cup D, V, f), C$ denotes an attribute set. $R \subseteq C$ and $X$ is a fuzzy set on $U . U / R=\left\{[x]_{1},[x]_{2}, \ldots,[x]_{l}\right\}$ is a partition space, where $[x]_{i}=\left\{x_{i_{1}}, x_{i_{2}}, \ldots, x_{i_{t_{i}}}\right\}(i=1,2, \ldots, l)$ and $t_{1}+t_{2}+\cdots+t_{l}=|U|, X$ is a fuzzy set on $U$. If $\mu_{X}\left(x_{i_{1}}\right)=$ $\mu_{X}\left(x_{i_{2}}\right)=\cdots=\mu_{X}\left(x_{i_{t_{i}}}\right)=c_{i}\left(0 \leq c_{i} \leq 1, i=1,2, \ldots, l\right)$, then $X$ is a step fuzzy set on $U / R$.

Definition 4 (average membership degree [40]). Given an information system $S=(U, C \cup D, V, f), R \subseteq C$ and $X$ is a fuzzy set on $U, U / R=\left\{[x]_{1},[x]_{2}, \ldots,[x]_{l}\right\}$ is a partition space. $\forall x \in[x]_{i}$, where $i=1,2, \ldots, l, \bar{\mu}(x)=$ $\bar{\mu}\left([x]_{i}\right)=\sum_{x \subseteq[x]_{i}} \mu(x) /\left|[x]_{i}\right|$, then we call that $\bar{\mu}(x)$ is the average membership degree.

In Definition $4, \bar{\mu}(x)$ is the average membership degree that an object $x \in[x]_{i}, i=1,2, \ldots, l$ belongs to the fuzzy concept $X .1-\bar{\mu}(x)$ is the average membership degree that an object $x \in[x]_{i}, i=1,2, \ldots, l$, belongs to the fuzzy concept $\bar{X}$. From the perspective of probability statistics, $\bar{\mu}(x)$ can be understood as the probability that an equivalent class $[x]_{i}, i=$ $1,2, \ldots, l$, belongs to the fuzzy concept $X$. Similarly, $1-\bar{\mu}(x)$ can be understood as the probability that an equivalent class $[x]_{i}, i=1,2, \ldots, l$, belongs to the fuzzy concept $\bar{X}$.

Definition 5 (average fuzzy set [41]). Given an information system $S=(U, C \cup D, V, f), R \subseteq C$ and $X$ is a fuzzy set on $U$, $U / R=\left\{[x]_{1},[x]_{2}, \ldots,[x]_{l}\right\}$ is a partition space, where $[x]_{i}=$ $\left\{x_{i_{1}}, x_{i_{2}}, \ldots, x_{i_{t_{i}}}\right\}(i=1,2, \ldots, l)$ and $t_{1}+t_{2}+\cdots+t_{l}=|U|, X$ is a fuzzy set on $U$. If $\mu_{X^{*}}\left(x_{i_{1}}\right)=\mu_{X^{*}}\left(x_{i_{2}}\right)=\cdots=\mu_{X^{*}}\left(x_{i_{i_{i}}}\right)=$ $\bar{\mu}\left([x]_{i}\right)=\sum_{x \subseteq[x]_{i}} \mu(x) /\left|[x]_{i}\right|, i=1,2, \ldots, l$, where $X^{*}$ is a fuzzy set on $U$, then we call $X^{*}$ is the average fuzzy set of $X$.

Example 6. Given an information system $S=(U, C \cup D, V, f)$, $R \subseteq C, X=0.1 / x_{1}+0.2 / x_{2}+0.5 / x_{3}+0.6 / x_{4}+1 / x_{5}+$ $1 / x_{6}+0.7 / x_{7}+0.3 / x_{8}+0.2 / x_{9}$ is a fuzzy set on $U ; U / R=$ $\left\{\left\{x_{1}, x_{2}\right\},\left\{x_{3}, x_{4}, x_{5}\right\},\left\{x_{6}, x_{7}\right\},\left\{x_{8}, x_{9}\right\}\right\}$, then

$$
\begin{aligned}
\underline{R}(X)= & \frac{0.1}{x_{1}}+\frac{0.1}{x_{2}}+\frac{0.5}{x_{3}}+\frac{0.5}{x_{4}}+\frac{0.5}{x_{5}}+\frac{0.7}{x_{6}}+\frac{0.7}{x_{7}} \\
& +\frac{0.2}{x_{8}}+\frac{0.2}{x_{9}}, \\
\bar{R}(X)= & \frac{0.2}{x_{1}}+\frac{0.2}{x_{2}}+\frac{1}{x_{3}}+\frac{1}{x_{4}}+\frac{1}{x_{5}}+\frac{1}{x_{6}}+\frac{1}{x_{7}}+\frac{0.3}{x_{8}} \\
& +\frac{0.3}{x_{9}}, \\
R\left(X^{*}\right)= & \frac{0.15}{x_{1}}+\frac{0.15}{x_{2}}+\frac{0.7}{x_{3}}+\frac{0.7}{x_{4}}+\frac{0.7}{x_{5}}+\frac{0.85}{x_{6}} \\
& +\frac{0.85}{x_{7}}+\frac{0.25}{x_{8}}+\frac{0.25}{x_{9}} .
\end{aligned}
$$

Definition 7 (probabilistic rough fuzzy sets [9]). Given an information system $S=(U, C \cup D, V, f)$ with a pair of threshold $\alpha, \beta(0 \leq \beta \leq \alpha \leq 1), R \subseteq C$ and $X$ is a fuzzy set on $U$, the lower and upper approximation sets of $X$ are defined as follows:

$$
\begin{aligned}
& \underline{R}^{(\alpha, \beta)}(X)=\{x \in U \mid \bar{\mu}([x]) \geq \alpha\}, \\
& \bar{R}^{(\alpha, \beta)}(X)=\{x \in U \mid \bar{\mu}([x])>\beta\} .
\end{aligned}
$$

Universe $U$ is divided by positive region, boundary region, and negative region, which can be defined as follows:

$$
\begin{aligned}
\operatorname{POS}_{R}^{(\alpha, \beta)}(X) & =\{x \in U \mid \bar{\mu}([x]) \geq \alpha\}=\underline{R}^{(\alpha, \beta)}(X), \\
\operatorname{BND}_{R}^{(\alpha, \beta)}(X) & =\{x \in U \mid \beta<\bar{\mu}([x])<\alpha\} \\
& =\bar{R}^{(\alpha, \beta)}(X)-\underline{R}^{(\alpha, \beta)}(X), \\
\operatorname{NEG}_{R}^{(\alpha, \beta)}(X) & =\{x \in U \mid \bar{\mu}([x]) \leq \beta\} \\
& =U-\bar{R}^{(\alpha, \beta)}(X) .
\end{aligned}
$$

Definition 8 (granularity measure $[21,22,42]$ ). Suppose $U$ is finite and nonempty universe. A function $m: 2^{U} \longrightarrow \mathfrak{R}$ is a granularity measure if it satisfies the following conditions for any $P, Q \in 2^{U}$ :
(1) $m(x) \geq 0$
(2) $P \subset Q \Longrightarrow m(P)<m(Q)$.
(3) $P \equiv_{s} Q \Longrightarrow m(P)=m(Q)$.

where $\equiv_{s}$ denotes there is a bijection from $P$ to $Q$.

Definition 9 (knowledge granulation [25]). Given an information system $S=(U, C \cup D, V, f), R \subseteq C$, and $X$ is a fuzzy set on $U, U / R=\left\{[x]_{1},[x]_{2}, \ldots,[x]_{l}\right\}$. The knowledge granulation of $U / R$ is defined as follows:

$$
G K(R)=\frac{\sum_{i=1}^{l}\left|[x]_{i}\right|^{2}}{|U|^{2}} .
$$

Obviously, $1 /|U| \leq G K(R) \leq 1$ for any $R \subseteq C$.

Definition 10 (fuzziness [43]). Suppose $U=\left(x_{1}, x_{2}, \ldots, x_{n}\right)$ be a finite universe, $A$ and $B$ be two fuzzy sets on $U$, if $H$ : $F(U) \longrightarrow[0,1]$ satisfies the following conditions:

(1) If and only if $A \in P(U), H(A)=0$.

(2) If and only if $\forall x_{i} \in U$ and $A\left(x_{i}\right)=1 / 2, H(A)=1$.

(3) $\forall x_{i} \in U$, if $B\left(x_{i}\right) \leq A\left(x_{i}\right) \leq 1 / 2$ or $B\left(x_{i}\right) \geq A\left(x_{i}\right) \geq$ $1 / 2$, then $H(B) \leq H(A)$.

(4) $\forall A \in F(U), H(A)=H(\bar{A})$.

where $H(\bullet)$ is called the fuzziness of a fuzzy subset.

Definition 11 (distance between two fuzzy sets [44]). A real function $d: F(U) \times F(U) \longrightarrow R^{+}$satisfies the following conditions: 
(1) $\forall A, B \in F(U), d(A, B)=d(B, A)$.

(2) $\forall A \in F(U), d(A, A)=0$.

(3) $\forall A \in F(U), d(A, \sim A)=\max _{\forall A, B \in F(U)} d(A, B)$.

(4) $\forall A, B, C \in F(U)$, if $A \subseteq B \subseteq C$, then $d(A, B) \leq$ $d(A, C)$ and $d(B, C) \leq d(A, C)$.

Then, $d(\bullet, \bullet)$ is a distance measurement.

\section{A Basic Fuzziness-Based Uncertainty Measure}

In the RFS model, the uncertainty usually comes from three regions at each granularity, because the objects in a positive or negative region are uncertain. Namely, the membership degrees of these objects may be not necessarily equal to 0 or 1 . With the adding attributes, the objects in the negative or positive regions may be reclassified and the three disjoint regions will be changed. As a result, the uncertainty at each granularity in RFS model will be changed accordingly. In this section, we will pay attention to analyze the change rules of the uncertainty of RFS in the changing knowledge spaces.

Research on uncertainty of RFS is very useful in approximate knowledge acquisition. Based on Definition 10, there are various fuzziness formulas proposed by many researchers. In this paper, we choose the fuzziness formula presented in [30] as follows:

$$
H_{R}(X)=\frac{4}{n} \sum_{i=1}^{n} \mu\left(x_{i}\right)\left(1-\mu\left(x_{i}\right)\right) .
$$

According to the analysis in Section 1, the uncertainty of a target concept in RFS comes from three regions: positive region, negative region. and boundary region; that is to say,

$$
\begin{aligned}
H_{R}(X)= & \frac{4}{n} \sum_{i=1}^{n} \bar{\mu}\left(x_{i}\right)\left(1-\bar{\mu}\left(x_{i}\right)\right) \\
= & \frac{4}{n} \sum_{x \in P O S_{R}^{(\alpha, \beta)}(X)} \bar{\mu}(x)(1-\bar{\mu}(x)) \\
& +\frac{4}{n} \sum_{x \in N E G_{R}^{(\alpha, \beta)}(X)} \bar{\mu}(x)(1-\bar{\mu}(x)) \\
& +\frac{4}{n} \sum_{x \in B N D_{R}^{(\alpha, \beta)}(X)} \bar{\mu}(x)(1-\bar{\mu}(x)) \\
= & H\left(P O S_{R}^{(\alpha, \beta)}(X)\right)+H\left(N E G_{R}^{(\alpha, \beta)}(X)\right) \\
& +H\left(B N D_{R}^{(\alpha, \beta)}(X)\right) .
\end{aligned}
$$

Obviously, given an information system $S=(U, C \cup$ $D, V, f), R \subseteq C$ and $X$ is a fuzzy set on $U$, the following properties hold for $H_{R}(X)$ :

(1) $0 \leq H_{R}(X) \leq 1$.

(2) $H_{R}(X)$ does not decrease with respect to $\alpha$ and does not increase with respect to $\beta$.
Therefore, the advantage of this method is independent of thresholds $(\alpha, \beta)$.

Lemma 12. Given an information system $S=(U, C \cup$ $D, V, f), R_{1} \subseteq R_{2} \subseteq C$. $X$ is a fuzzy set on $U, U / R_{1}=$ $\left\{p_{1}, p_{2}, \ldots, p_{l}\right\}$ and $U / R_{2}=\left\{q_{1}, q_{2}, \ldots, q_{m}\right\}$. If a granule $p_{i}(i=1,2, \ldots, l)$ which is subdivided into many finer subgranules $q_{i_{1}}, q_{i_{2}}, \ldots, q_{i_{m_{i}}}$ by $\Delta R=R_{2}-R_{1}$, and $\bar{\mu}\left(q_{i_{1}}\right)=$ $\bar{\mu}\left(q_{i_{2}}\right)=\cdots=\bar{\mu}\left(q_{i_{m_{i}}}\right)=\mu$, then $\bar{\mu}\left(p_{i}\right)=\mu$.

Proof. Obviously, $\bar{\mu}\left(p_{i}\right)=\left(\left|q_{i_{1}}\right| /\left|p_{i}\right|\right) \bar{\mu}\left(q_{i_{1}}\right)+\left(\left|q_{i_{2}}\right| /\left|p_{i}\right|\right) \bar{\mu}\left(q_{i_{2}}\right)+$ $\cdots+\left(\left|q_{i_{m_{i}}}\right| /\left|p_{i}\right|\right) \bar{\mu}\left(q_{i_{m_{i}}}\right)$, if $\bar{\mu}\left(q_{i_{1}}\right)=\bar{\mu}\left(q_{i_{2}}\right)=\cdots=\bar{\mu}\left(q_{i_{m_{i}}}\right)=$ $\mu_{i}$, because $\left|p_{i}\right|=\left|q_{i_{1}}\right|+\left|q_{i_{2}}\right|+\cdots+\left|q_{i_{m_{i}}}\right|$, then $\bar{\mu}\left(p_{i}\right)=$ $\left(\left|q_{i_{1}}\right| /\left|p_{i}\right|\right) \mu+\left(\left|q_{i_{2}}\right| /\left|p_{i}\right|\right) \mu+\cdots+\left(\left|q_{i_{m_{i}}}\right| /\left|p_{i}\right|\right) \mu$.

From Lemma 12, if the granule on the coarser granularity is subdivided into many finer subgranules with the same membership degree on the finer granularity, then the membership degree of the equivalence class is equal to the one of its subequivalence classes.

Theorem 13. Given an information system $S=(U, C \cup$ $D, V, f), R_{1} \subseteq R_{2} \subseteq C$. $X$ is a fuzzy set on $U, U / R_{1}=$ $\left\{p_{1}, p_{2}, \ldots, p_{l}\right\}$ and $U / R_{2}=\left\{q_{1}, q_{2}, \ldots, q_{m}\right\}$. If only granule $p_{i}(i=1,2, \ldots, l)$ is subdivided into many finer subgranules $q_{i_{1}}, q_{i_{2}}, \ldots, q_{i_{m_{i}}}$ by $\Delta R=R_{2}-R_{1}$, and $\bar{\mu}\left(q_{i_{1}}\right)=\bar{\mu}\left(q_{i_{2}}\right)=\cdots=$ $\bar{\mu}\left(q_{i_{m_{i}}}\right)$, then $H_{R_{1}}(X)=H_{R_{2}}(X)$.

Proof. From Lemma 12, Theorem 13 is easy to prove.

From Theorem 13, we can conclude that the uncertainty will remain unchanged if the granules on the coarser granularity which are subdivided into many finer subgranules by equal proportion on the finer granularity.

Theorem 14. Given information system $S=(U, C \cup D, V, f)$, $R_{1} \subseteq C$ and $R_{2} \subseteq C$. $X$ is a fuzzy set on $U$. If $R_{1} \subseteq$ $R_{2}, H_{R_{1}}(X) \geq H_{R_{2}}(X)$ holds.

Proof. Let $U=\left\{x_{1}, x_{2}, \ldots, x_{n}\right\}$ be a nonempty finite domain, $U / R_{1}=\left\{p_{1}, p_{2}, \ldots, p_{l}\right\}$ and $U / R_{2}=\left\{q_{1}, q_{2}, \ldots, q_{m}\right\}$. Because $R_{1} \subseteq R_{2}, U / R_{2} \preceq U / R_{1}$. According to the conditions, for simplicity, suppose only one granule $p_{1}$ can be subdivided into two finer subgranules (the more complicated cases can be transformed into this case, so we will not repeat them here). Without loss of generality, let $p_{1}=q_{1} \cup q_{2}, p_{2}=$ $q_{3}, p_{3}=q_{4}, p_{l}=q_{m}(m=l+1)$; namely, $U / R_{2}=$ $\left\{q_{1}, q_{2}, p_{2}, p_{3}, \ldots, p_{m}\right\}$. We will prove this theorem in the following:

$$
\begin{aligned}
& \Delta H_{R_{1}-R_{2}}=H_{R_{1}}(X)-H_{R_{2}}(X) \\
& \quad=\frac{4}{n}\left(\bar{\mu}\left(p_{1}\right)\left(1-\bar{\mu}\left(p_{1}\right)\right)\left|p_{1}\right|\right. \\
& \left.\quad-\bar{\mu}\left(q_{1}\right)\left(1-\bar{\mu}\left(q_{1}\right)\right)\left|q_{1}\right|-\bar{\mu}\left(q_{2}\right)\left(1-\bar{\mu}\left(q_{2}\right)\right)\left|q_{2}\right|\right) \\
& \quad=\frac{4}{n}\left(\left|q_{1}\right| \bar{\mu}\left(q_{1}\right)^{2}+\left|q_{2}\right| \bar{\mu}\left(q_{2}\right)^{2}-\left|p_{1}\right| \bar{\mu}\left(p_{1}\right)^{2}\right) .
\end{aligned}
$$


Because $\bar{\mu}\left(p_{1}\right)=\left(\left|q_{1}\right| /\left|p_{1}\right|\right) \bar{\mu}\left(q_{1}\right)+\left(\left|q_{2}\right| /\left|p_{1}\right|\right) \bar{\mu}\left(q_{2}\right), \bar{\mu}\left(p_{1}\right)^{2}=$ $\left(\left|q_{1}\right|^{2} /\left|p_{1}\right|^{2}\right) \bar{\mu}\left(q_{1}\right)^{2}+2\left(\left|q_{1}\right|\left|q_{2}\right| /\left|p_{1}\right|^{2}\right) \bar{\mu}\left(q_{1}\right) \bar{\mu}\left(q_{2}\right)+\left(\left|q_{2}\right|^{2} /\right.$ $\left.\left|p_{1}\right|^{2}\right) \bar{\mu}\left(q_{2}\right)^{2}$.

We have

$$
\begin{aligned}
& \Delta H_{R_{1}-R_{2}} \\
& \quad=\frac{4\left|q_{1}\right|\left|q_{2}\right|}{n\left|p_{1}\right|^{2}}\left(\bar{\mu}\left(q_{1}\right)^{2}-2 \bar{\mu}\left(q_{1}\right) \bar{\mu}\left(q_{2}\right)+\bar{\mu}\left(q_{2}\right)^{2}\right) \\
& \quad=\frac{4\left|q_{1}\right|\left|q_{2}\right|}{n\left|p_{1}\right|^{2}}\left(\bar{\mu}\left(q_{1}\right)-\bar{\mu}\left(q_{2}\right)\right)^{2} \geq 0 .
\end{aligned}
$$

Thus, $H_{R_{1}}(X) \geq H_{R_{2}}(X)$, where $H_{R_{1}}(X)=H_{R_{2}}(X)$ when $\bar{\mu}\left(q_{1}\right)=\bar{\mu}\left(q_{2}\right)$.

From Theorem 14, we know that the total uncertainty will monotonically decrease with finer granularity in RFS model.

Theorem 15. Given an information system $S=(U, C \cup$ $D, V, f)$, for any $0 \leq \beta \leq \alpha \leq 1, R_{1} \subseteq C$ and $R_{2} \subseteq C$. $X$ is a fuzzy set on $U$. If $R_{1} \subseteq R_{2}$ and only the granules containing in $\operatorname{POS}_{R_{1}}^{(\alpha, \beta)}(X)$ are subdivided into many finer equivalence classes by the attribute increment $\Delta R=R_{2}-R_{1}$, then $H\left(B N D_{R_{1}}^{(\alpha, \beta)}(X)\right) \leq H\left(B N D_{R_{2}}^{(\alpha, \beta)}(X)\right)$ hold.

Proof. Let $U=\left\{x_{1}, x_{2}, \ldots, x_{n}\right\}$ be a nonempty finite domain, $U / R_{1}=\left\{p_{1}, p_{2}, \ldots, p_{l}\right\}$, because $R_{1} \subseteq R_{2}, U / R_{2} \leq U / R_{1}$. According to the conditions, for simplicity, suppose only one granule $p_{1}$ can be subdivided into two finer subgranules by $\Delta R=R_{2}-R_{1}$ (the more complicated cases can be transformed into this case, so we will not repeat them here). Without loss of generality, let $p_{1}=q_{1} \cup q_{2}, p_{2}=q_{3}, p_{3}=q_{4}, p_{l}=q_{m}(m=$ $l+1)$; namely, $U / R_{1}=\left\{q_{1}, q_{2}, p_{2}, p_{3}, \ldots, p_{l}\right\}$. We will prove this theorem in three cases in the following.

Case 1. If $\bar{\mu}\left(q_{1}\right) \leq \beta$ and $\bar{\mu}\left(q_{2}\right) \leq \beta$, namely, $q_{1} \subseteq N E G_{R_{2}}^{(\alpha, \beta)}(X)$ and $q_{2} \subseteq N E G_{R_{2}}^{(\alpha, \beta)}(X), B N D_{R_{1}}^{\left(\alpha_{1}, \beta_{1}\right)}(X)=B N D_{R_{2}}^{\left(\alpha_{2}, \beta_{2}\right)}(X)$. Because $p_{2}=q_{3}, p_{3}=q_{4}, p_{l}=q_{m}(m=l+1)$, $H\left(B N D_{R_{1}}^{(\alpha, \beta)}(X)\right)=H\left(B N D_{R_{2}}^{(\alpha, \beta)}(X)\right)$.

Case 2. If $\bar{\mu}\left(q_{1}\right) \geq \alpha$ and $\bar{\mu}\left(q_{2}\right) \leq \beta$, namely, $q_{1} \subseteq \operatorname{POS}_{R_{2}}^{(\alpha, \beta)}(X)$ and $q_{2} \subseteq N E G_{R_{2}}^{(\alpha, \beta)}(X), B N D_{R_{1}}^{\left(\alpha_{1}, \beta_{1}\right)}(X)=B N D_{R_{2}}^{\left(\alpha_{2}, \beta_{2}\right)}(X)$. Because $p_{2}=q_{3}, p_{3}=q_{4}, p_{l}=q_{m}(m=l+1)$, $H\left(B N D_{R_{1}}^{(\alpha, \beta)}(X)\right)=H\left(B N D_{R_{2}}^{(\alpha, \beta)}(X)\right)$.

Case 3. If $\beta<\bar{\mu}\left(q_{1}\right)<\alpha$ and $\bar{\mu}\left(q_{2}\right) \leq \beta$, namely, $q_{1} \subseteq$ $B N D_{R_{2}}^{(\alpha, \beta)}(X)$ and $q_{2} \subseteq N E G_{R_{2}}^{(\alpha, \beta)}(X)$, then $B N D_{R_{2}}^{\left(\alpha_{1}, \beta_{1}\right)}(X)=$ $B N D_{R_{1}}^{\left(\alpha_{2}, \beta_{2}\right)}(X) \cup q_{1}, H\left(B N D_{R_{2}}^{(\alpha, \beta)}(X)\right)=H\left(B N D_{R_{1}}^{(\alpha, \beta)}(X)\right)+$ $H\left(q_{1}\right)>H\left(B N D_{R_{2}}^{(\alpha, \beta)}(X)\right)$.

Theorem 16. Given an information system $S=(U, C \cup$ $D, V, f)$, for any $0 \leq \beta \leq \alpha \leq 1, R_{1} \subseteq C$ and $R_{2} \subseteq C$. $X$ is a fuzzy set on $U$. If $R_{1} \subseteq R_{2}$ and only the granules containing in $N E G_{R_{1}}^{(\alpha, \beta)}(X)$ are subdivided into many finer equivalence classes by the attribute increment $\Delta R=R_{2}-R_{1}$, then $H\left(B N D_{R_{1}}^{(\alpha, \beta)}(X)\right) \leq H\left(B N D_{R_{2}}^{(\alpha, \beta)}(X)\right)$ hold.

Proof. Similar to Theorem 15, Theorem 16 is easy to prove.

According to Theorems 15 and 16, the uncertainty of boundary region will increase only when the equivalence classes contained in negative region or positive region are subdivided with finer granularity in RFS model. This is not in line with human cognitive habits.

Theorem 17. Given an information system $S=(U, C \cup$ $D, V, f)$, for any $0 \leq \beta \leq \alpha \leq 1, R_{1} \subseteq C$ and $R_{2} \subseteq C$. $X$ is a fuzzy set on $U$. If $R_{1} \subseteq R_{2}$ and only the granules containing in $B N D_{R_{1}}^{(\alpha, \beta)}(X)$ are subdivided into many finer equivalence classes by the attribute increment $\Delta R=R_{2}-R_{1}$, then $H\left(B N D_{R_{1}}^{(\alpha, \beta)}(X)\right) \geq H\left(B N D_{R_{2}}^{(\alpha, \beta)}(X)\right)$ hold.

Proof. Let $U=\left\{x_{1}, x_{2}, \ldots, x_{n}\right\}$ be a nonempty finite domain, $U / R_{1}=\left\{p_{1}, p_{2}, \ldots, p_{l}\right\}$ and $U / R_{2}=\left\{q_{1}, q_{2}, \ldots, q_{m}\right\}$. Because $R_{1} \subseteq R_{2}, U / R_{2} \leq U / R_{1}$. According to the condition, for simplicity, suppose only one granule $p_{1}$ can be subdivided into two finer subgranules by $\Delta R=R_{2}-R_{1}$ (the more complicated cases can be transformed into this case, so we will not repeat them here). Without loss of generality, let $p_{1}=q_{1} \cup q_{2}, p_{2}=q_{3}, p_{3}=q_{4}, p_{l}=q_{m}(m=l+1)$, namely, $U / R_{1}=\left\{q_{1}, q_{2}, p_{2}, p_{3}, \ldots, p_{l}\right\}$. We will prove this theorem in four cases in the following.

Case 1. If $\beta<\bar{\mu}\left(q_{1}\right)<\alpha$ and $\beta<\bar{\mu}\left(q_{2}\right)<\alpha$, namely, $q_{1} \subseteq B N D_{R_{2}}^{(\alpha, \beta)}(X)$ and $q_{2} \subseteq B N D_{R_{2}}^{(\alpha, \beta)}(X), B N D_{R_{1}}^{\left(\alpha_{1}, \beta_{1}\right)}(X)=$ $B N D_{R_{2}}^{\left(\alpha_{2}, \beta_{2}\right)}(X)$. Because $p_{2}=q_{3}, p_{3}=q_{4}, p_{l}=q_{m}(m=l+1)$, $H\left(B N D_{R_{1}}^{(\alpha, \beta)}(X)\right)=H\left(B N D_{R_{2}}^{(\alpha, \beta)}(X)\right)$.

Case 2. If $\bar{\mu}\left(q_{1}\right) \geq \alpha$ and $\bar{\mu}\left(q_{2}\right) \leq \beta$, namely, $q_{1} \subseteq \operatorname{POS}_{R_{2}}^{(\alpha, \beta)}(X)$ and $q_{2} \subseteq N E G_{R_{2}}^{(\alpha, \beta)}(X), B N D_{R_{1}}^{\left(\alpha_{1}, \beta_{1}\right)}(X)>B N D_{R_{2}}^{\left(\alpha_{2}, \beta_{2}\right)}(X)$. Because $p_{2}=q_{3}, p_{3}=q_{4}, p_{l}=q_{m}(m=l+1)$, $H\left(B N D_{R_{1}}^{(\alpha, \beta)}(X)\right)>H\left(B N D_{R_{2}}^{(\alpha, \beta)}(X)\right)$.

Case 3. If $\beta<\bar{\mu}\left(q_{1}\right)<\alpha$ and $\bar{\mu}\left(q_{2}\right) \geq \alpha$, namely, $q_{1}$ $\subseteq B N D_{R_{2}}^{(\alpha, \beta)}(X)$ and $q_{2} \subseteq \operatorname{POS}_{R_{2}}^{(\alpha, \beta)}(X), B N D_{R_{1}}^{\left(\alpha_{1}, \beta_{1}\right)}(X)>$ $B N D_{R_{2}}^{\left(\alpha_{2}, \beta_{2}\right)}(X)$. Because $p_{2}=q_{3}, p_{3}=q_{4}, p_{l}=q_{m}(m=l+1)$, $H\left(B N D_{R_{1}}^{(\alpha, \beta)}(X)\right)>H\left(B N D_{R_{2}}^{(\alpha, \beta)}(X)\right)$.

Case 4. $\beta<\bar{\mu}\left(q_{1}\right)<\alpha$ and $\bar{\mu}\left(q_{2}\right) \leq \beta$, namely, $q_{1} \subseteq$ $B N D_{R_{2}}^{(\alpha, \beta)}(X)$ and $q_{2} \subseteq N E G_{R_{2}}^{(\alpha, \beta)}(X), B N D_{R_{1}}^{\left(\alpha_{1}, \beta_{1}\right)}(X)>$ $B N D_{R_{2}}^{\left(\alpha_{2}, \beta_{2}\right)}(X)$. Because $p_{2}=q_{3}, p_{3}=q_{4}, p_{l}=q_{m}(m=l+1)$, $H\left(B N D_{R_{1}}^{(\alpha, \beta)}(X)\right)>H\left(B N D_{R_{2}}^{(\alpha, \beta)}(X)\right)$.

According to Theorem 17, the uncertainty of boundary region will decrease when only the equivalence classes contained in boundary region are subdivided with finer 
granularity in RFS model. This is in line with human cognitive habits.

Theorem 18. Given an information system $S=(U, C \cup$ $D, V, f), R \subseteq C, X$ is a fuzzy set on $U$. If $0 \leq \beta_{1} \leq \beta_{2} \leq$ $\alpha_{2} \leq \alpha_{1} \leq 1$, then the following properties hold:

(1) $H\left(B N D_{R}^{\left(\alpha_{1}, \beta_{1}\right)}(X)\right) \geq H\left(B N D_{R}^{\left(\alpha_{2}, \beta_{2}\right)}(X)\right)$.

(2) $H\left(\operatorname{POS}_{R}^{\left(\alpha_{2}, \beta_{1}\right)}(X)\right) \geq H\left(\operatorname{POS}_{R}^{\left(\alpha_{1}, \beta_{1}\right)}(X)\right)$.

(3) $H\left(N E G_{R}^{\left(\alpha_{1}, \beta_{2}\right)}(X)\right) \geq H\left(N E G_{R}^{\left(\alpha_{1}, \beta_{1}\right)}(X)\right)$.

Proof. Obviously, some of the equivalence classes in positive region will be assigned to the boundary region as parameter $\alpha$ increases, so $H\left(\operatorname{POS}_{R}^{(\alpha, \beta)}(X)\right)$ will decrease. Similarly, some of the equivalence classes in negative region will be assigned to the boundary region as parameter $\beta$ decreases, so $H\left(N E G_{R}^{(\alpha, \beta)}(X)\right)$ will decrease. Some of the equivalence classes in boundary region will be assigned to the negative region or positive region as parameter $\alpha$ increases and parameter $\beta$ decreases, so $H\left(B N D_{R}^{(\alpha, \beta)}(X)\right)$ will decrease.

Theorem 18 indicates that the uncertainty of positive region monotonically decreases as parameter $\alpha$ increases, the uncertainty of negative region monotonically decreases as parameter $\beta$ decreases, and uncertainty of boundary region monotonically increases with increasing $\alpha$ and decreasing $\beta$.

Note. As to the monotonicity of $H_{R}(X)$, it can be used for measuring uncertainty in RFS to a certain degree. However, there still exist some deficiencies about $H_{R}(X)$; for example, it cannot provide enough information, which will be discussed in the following section.

\section{Modified Uncertainty Measure of Rough Fuzzy Sets}

According to the above discussion, $H_{R}(X)$ is monotonic with finer approximation spaces in a hierarchical granular structure, which can reflect the change rules of uncertainty in RFS to a certain extent. However, there exist some deficiencies about $H_{R}(X)$. On the one hand, $H_{R}(X)$ does not provide enough information that is caused by the uncertainty related to the granularity of partitions. This limitation will be revealed by Example 19 in this section. One the other hand, $H_{R}(X)$ cannot supply enough information to characterize the two approximation spaces with the same fuzziness. In particualr, the latter is very important for evaluating the attribute significance in attribute reduct.

Example 19. Given an information system $S=(U, C \cup$ $D, V, f)$, where $U=\left\{x_{1}, x_{2}, x_{3}, x_{4}, x_{5}, x_{6}, x_{7}\right\}, R_{1}, R_{2} \subseteq$ $C$ and $X=0.3 / x_{1}+0.5 / x_{2}+0.7 / x_{3}+0.9 / x_{4}+$ $0.8 / x_{5}+0.5 / x_{6}+0.2 / x_{7}$ is a fuzzy set on $U$. Suppose that $U / R_{1}=\left\{\left\{x_{1}, x_{2}, x_{3}\right\},\left\{x_{4}, x_{5}, x_{6}, x_{7}\right\}\right\}, U / R_{2}=$ $\left\{\left\{x_{2}\right\},\left\{x_{1}, x_{3}\right\},\left\{x_{4}, x_{5}, x_{6}, x_{7}\right\}\right\}$, and $U / C=\left\{\left\{x_{2}\right\},\left\{x_{1}, x_{3}\right\}\right.$, $\left.\left\{x_{4}, x_{5}\right\},\left\{x_{6}, x_{7}\right\}\right\}$.
According to formula (8), we have

$$
H_{R_{1}}(X)=H_{R_{2}}(X)=0.977 \text {. }
$$

Therefore, $H_{R}(X)$ cannot reflect the uncertainty caused by the granularity of partition.

To overcome this limitation, we modify formula (8) based on the idea of combination entropy and combination granulation $[42,45]$, and the definition of combined fuzzinessbased uncertainty measure is as follows.

Definition 20. Given an information system $S=(U, C \cup$ $D, V, f)$, and $X$ is a fuzzy set on $U$. The combined fuzzinessbased uncertainty measure is defined as follows:

$$
C H_{R}(X)=H_{R}(X) * G K(R) .
$$

Theorem 21 (see [25]). Given an information system $S=$ $(U, C \cup D, V, f)$, and $X$ is a fuzzy set on $U$. If $R_{1} \subset R_{2} \subseteq$ $C, G K\left(R_{1}\right)>G K\left(R_{2}\right)$ holds.

Theorem 22. Given an information system $S=(U, C \cup$ $D, V, f), X$ is a fuzzy set on $U$. If $R_{1} \subset R_{2} \subseteq C, C H_{R_{1}}(X)>$ $\mathrm{CH}_{\mathrm{R}_{2}}(\mathrm{X})$ holds.

Proof. If $R_{1} \subset R_{2} \subseteq C$, we have $G K\left(R_{1}\right)>G K\left(R_{2}\right)$ and $H_{R_{1}}(X) \geq H_{R_{2}}(X)$ by Theorem 21 and Theorem 14, respectively. Then, we obtain $\mathrm{CH}_{R_{1}}(X)>\mathrm{CH}_{R_{2}}(X)$.

According to Theorem 22, the combined fuzziness-based uncertainty in Definition 20 is more accurate than the basic fuzziness-based uncertainty, since it is equipped with strict monotonicity.

Example 19 (continued). We have

$$
\begin{aligned}
& C H_{R_{1}}(X)=0.498, \\
& C H_{R_{2}}(X)=0.418 .
\end{aligned}
$$

Obviously, $\mathrm{CH}_{R_{1}}(\mathrm{X})>\mathrm{CH}_{\mathrm{R}_{2}}(X)$.

Thus, $U / R_{2} \prec U / R_{1} \Longrightarrow C H_{R_{2}}(X)<C H_{R_{1}}(X)$.

In some special cases, the two rough approximation spaces with the same uncertainty are not necessarily equal. Consequently, it is hard to distinguish them, which is important to evaluate the attribute significance in attribute reduction. Therefore, it is necessary to further modify formula (13). Firstly, based on Definition 11, we have the following definition.

Definition 23 (fuzzy distance). Given an information system $S=(U, C \cup D, V, f), R_{1}, R_{2} \subseteq C$, and $X$ is a fuzzy set on $U$. $U / R_{1}=\left\{p_{1}, p_{2}, \ldots, p_{l}\right\}$ and $U / R_{2}=\left\{q_{1}, q_{2}, \ldots, q_{m}\right\}$ are two rough approximation spaces on $X$. Then, the fuzzy distance between $U / R_{1}$ and $U / R_{2}$ is defined as follows:

$$
D\left(\frac{U}{R_{1}}, \frac{U}{R_{2}}\right)=\frac{1}{2}\left(D_{1}\left(\frac{U}{R_{1}}, \frac{U}{R_{2}}\right)+D_{2}\left(\frac{U}{R_{1}}, \frac{U}{R_{2}}\right)\right) \text {, }
$$

where

$$
D_{1}\left(\frac{U}{R_{1}}, \frac{U}{R_{2}}\right)=\frac{1}{\sqrt{n}}\left(\sum_{i=1}^{n}\left(\mu_{\underline{R}_{1}}\left(x_{i}\right)-\mu_{\underline{R}_{2}}\left(x_{i}\right)\right)^{2}\right)^{1 / 2}
$$


and

$$
D_{2}\left(\frac{U}{R_{1}}, \frac{U}{R_{2}}\right)=\frac{1}{\sqrt{n}}\left(\sum_{i=1}^{n}\left(\mu_{\bar{R}_{1}}\left(x_{i}\right)-\mu_{\bar{R}_{2}}\left(x_{i}\right)\right)^{2}\right)^{1 / 2} .
$$

Theorem 24. Given an information system $S=(U, C \cup$ $D, V, f), R_{1} \subseteq C, R_{2} \subseteq C$, and $R_{3} \subseteq C$, and $X$ is a fuzzy set on $U$. If $R_{1} \subseteq R_{2} \subseteq R_{3}, D\left(U / R_{1}, U / R_{2}\right) \leq D\left(U / R_{1}, U / R_{3}\right)$ holds.

Proof. Let $U=\left\{x_{1}, x_{2}, \ldots, x_{n}\right\}$ be a nonempty finite universe; $U / R_{1}=\left\{p_{1}, p_{2}, \ldots, p_{l}\right\}, U / R_{2}=\left\{q_{1}, q_{2}, \ldots, q_{m}\right\}$, and $U / R_{3}=$ $\left\{r_{1}, r_{2}, \ldots, r_{s}\right\}$. Because $R_{1} \subseteq R_{2} \subseteq R_{3}, U / R_{1} \preceq U / R_{2} \preceq U / R_{3}$. According to the condition, for simplicity, suppose only one granule $p_{1}$ can be subdivided into two finer subgranules and $q_{2}$ by $\Delta R=R_{2}-R_{1}$ and only one granule $q_{1}$ can be subdivided into two finer subgranules $r_{1}$ and $r_{2}$ by $\Delta R=R_{3}-R_{2}$ (the more complicated cases can be transformed into this case, so we will not repeat them here). Without loss of generality, let $p_{1}=q_{1} \cup q_{2}, p_{2}=q_{3}, p_{3}=q_{4}, \ldots, p_{l}=q_{m}(m=l+1), q_{1}=$ $r_{1} \cup r_{2}, q_{2}=r_{3}, q_{3}=r_{4}, \ldots, q_{n}=r_{s}(s=m+1)$, namely, $U / R_{1}=\left\{q_{1}, q_{2}, p_{2}, p_{3}, \ldots, p_{l}\right\}, U / R_{2}=\left\{r_{1}, r_{2}, q_{2}, q_{3}, \ldots, q_{m}\right\}$. Then, we have

$$
\begin{aligned}
& D_{1}\left(\frac{U}{R_{1}}, \frac{U}{R_{2}}\right)=\frac{1}{\sqrt{n}}\left(\sum_{i=1}^{n}\left(\mu_{\underline{R}_{1}}\left(x_{i}\right)-\mu_{\underline{R}_{2}}\left(x_{i}\right)\right)^{2}\right)^{1 / 2} \\
& \quad=\frac{1}{\sqrt{n}}\left(\left(\mu_{\underline{R}_{1}}\left(p_{1}\right)-\mu_{\underline{R}_{2}}\left(q_{1}\right)\right)^{2}\left|q_{1}\right|\right. \\
& \left.\quad+\left(\mu_{\underline{R}_{1}}\left(p_{1}\right)-\mu_{\underline{R}_{2}}\left(q_{2}\right)\right)^{2}\left|q_{2}\right|\right)^{1 / 2}, \\
& D_{1}\left(\frac{U}{R_{1}}, \frac{U}{R_{3}}\right)=\frac{1}{\sqrt{n}}\left(\sum_{i=1}^{n}\left(\mu_{\underline{R}_{1}}\left(x_{i}\right)-\mu_{\underline{R}_{3}}\left(x_{i}\right)\right)^{2}\right)^{1 / 2} \\
& \quad=\frac{1}{\sqrt{n}}\left(\left(\mu_{\underline{R}_{1}}\left(p_{1}\right)-\mu_{\underline{R}_{3}}\left(r_{1}\right)\right)^{2}\left|r_{1}\right|\right. \\
& \quad+\left(\mu_{\underline{R}_{1}}\left(p_{1}\right)-\mu_{\underline{R}_{3}}\left(r_{2}\right)\right)^{2}\left|r_{2}\right| \\
& \left.\quad+\left(\mu_{\underline{R}_{1}}\left(p_{1}\right)-\mu_{\underline{R}_{3}}\left(r_{3}\right)\right)^{2}\left|r_{3}\right|\right)^{1 / 2} .
\end{aligned}
$$

Because $q_{2}=r_{3},\left(\mu_{\underline{R}_{1}}\left(p_{1}\right)-\mu_{\underline{R}_{2}}\left(q_{2}\right)\right)^{2}\left|q_{2}\right|=\left(\mu_{\underline{R}_{1}}\left(p_{1}\right)-\right.$ $\left.\mu_{\underline{R}_{3}}\left(r_{3}\right)\right)^{2}\left|r_{3}\right|$.

From Definition 2, $\mu_{\underline{R}_{1}}\left(p_{1}\right)-\mu_{\underline{R}_{2}}\left(q_{1}\right) \leq \mu_{\underline{R}_{1}}\left(p_{1}\right)-\mu_{\underline{R}_{3}}\left(r_{1}\right)$ and $\mu_{\underline{R}_{1}}\left(p_{1}\right)-\mu_{\underline{R}_{2}}\left(q_{1}\right) \leq \bar{\mu}_{\underline{R}_{1}}\left(p_{1}\right)-\underline{\mu}_{\underline{R}_{3}}\left(r_{2}\right)$.

Because $q_{1}=r_{1} \cup r_{2},\left(\mu_{\underline{R}_{1}}\left(p_{1}\right)-\mu_{\underline{R}_{2}}\left(q_{1}\right)\right)^{2}\left|q_{1}\right| \leq\left(\mu_{\underline{R}_{1}}\left(p_{1}\right)-\right.$ $\left.\mu_{\underline{R}_{3}}\left(r_{1}\right)\right)^{2}\left|r_{1}\right|+\left(\mu_{\underline{R}_{1}}\left(p_{1}\right)-\mu_{\underline{R}_{3}}\left(r_{2}\right)\right)^{2}\left|r_{2}\right|$.

Thus, $\Delta D=D_{1}\left(U / R_{1}, \bar{U} / R_{3}\right)-D_{1}\left(U / R_{1}, U / R_{2}\right) \geq 0$.

Then, we have

$$
D_{1}\left(\frac{U}{R_{1}}, \frac{U}{R_{2}}\right) \leq D_{1}\left(\frac{U}{R_{1}}, \frac{U}{R_{3}}\right) .
$$

Similarly, one can draw

$$
D_{2}\left(\frac{U}{R_{1}}, \frac{U}{R_{2}}\right) \leq D_{2}\left(\frac{U}{R_{1}}, \frac{U}{R_{3}}\right) .
$$

Therefore, we have

$$
D\left(\frac{U}{R_{2}}, \frac{U}{R_{3}}\right) \leq D\left(\frac{U}{R_{1}}, \frac{U}{R_{3}}\right) .
$$

From Theorem 24, we can easily find that formula (15) in Definition 23 is a distance measurement between two fuzzy sets, because it satisfies all conditions of Definition 11. Thus, formula (15) is able to characterize the difference between two rough approximation spaces. Then, we have the definition of the improved uncertainty measure as follows.

Definition 25. Given an information system $S=(U, C \cup$ $D, V, f), R_{1} \subseteq C$ and $R_{2} \subseteq C$, and $X$ is a fuzzy set on $U$. The improved fuzziness-based uncertainty measure is defined as follows:

$$
U N C_{R}(X)=\frac{1}{2}\left(C_{R}(X)+D\left(\frac{U}{R}, \frac{U}{C}\right)\right) .
$$

Theorem 26. Given an information system $S=(U, C \cup D$, $V, f)$, and $X$ is a fuzzy set on $U$. If $R_{1} \subset R_{2} \subseteq C, U N C_{R_{1}}(X)>$ $U N C_{R_{2}}(X)$ holds.

Proof. If $U / R_{2} \prec U / R_{1}$, we have $C H_{R_{1}}(X)>C H_{R_{2}}(X)$ by Theorem 22. According to Theorem 24 , we have $D\left(U / R_{1}\right.$, $U / C) \geq D\left(U / R_{2}, U / C\right)$. Then, we obtain $U N C_{R_{1}}(X)>$ $U N C_{R_{2}}(X)$.

From Theorem 26, we learn that the modified fuzzinessbased uncertainty in Definition 25 is also strictly monotonic with finer granularity.

Theorem 27. Given an information system $S=(U, C \cup$ $D, V, f), R \subseteq C$, and $X$ is a fuzzy set on $U$. Then, $0 \leq$ $\operatorname{UNC}_{R}^{(\alpha, \beta)}(X) \leq 1$.

Example 19(continued). We have

$$
\begin{aligned}
& D_{1}\left(\frac{U}{R_{1}}, \frac{U}{C}\right)=0.63 \\
& D_{1}\left(\frac{U}{R_{2}}, \frac{U}{C}\right)=0.6 \\
& D_{2}\left(\frac{U}{R_{1}}, \frac{U}{C}\right)=0.45 \\
& D_{2}\left(\frac{U}{R_{2}}, \frac{U}{C}\right)=0.4 \\
& D\left(\frac{U}{R_{1}}, \frac{U}{C}\right)=0.54 \\
& D\left(\frac{U}{R_{2}}, \frac{U}{C}\right)=0.5 \\
& U N C_{R_{1}}(X)=\frac{1}{2}(0.498+0.54)=0.519 \\
& U N C_{R_{2}}(X)=\frac{1}{2}(0.418+0.5)=0.459
\end{aligned}
$$

Obviously, $U N C_{R_{1}}(X)>U N C_{R_{2}}(X)$. 
Thus, $U / R_{2} \prec U / R_{1} \Longrightarrow U N C_{R_{2}}(X)>U N C_{R_{1}}(X)$.

Theorem 28. Given an information system $S=(U, C \cup$ $D, V, f)$, for any $0 \leq \beta \leq \alpha \leq 1, R \subseteq C, X$ is a fuzzy set on $U$. If $0 \leq \beta_{1} \leq \beta_{2} \leq \alpha_{2} \leq \alpha_{1} \leq 1$, then the following properties hold:

(1) $\operatorname{UNC}\left(B N D_{R}^{\left(\alpha_{1}, \beta_{1}\right)}(X)\right) \geq \operatorname{UNC}\left(B N D_{R}^{\left(\alpha_{2}, \beta_{2}\right)}(X)\right)$.

(2) $\operatorname{UNC}\left(\operatorname{POS}_{R}^{\left(\alpha_{2}, \beta_{1}\right)}(X)\right) \geq U N C\left(P O S_{R}^{\left(\alpha_{1}, \beta_{1}\right)}(X)\right)$.

(3) $\operatorname{UNC}\left(N E G_{R}^{\left(\alpha_{1}, \beta_{2}\right)}(X)\right) \geq \operatorname{UNC}\left(N E G_{R}^{\left(\alpha_{1}, \beta_{1}\right)}(X)\right)$.

Proof. Because $G K(R)$ and $D(U / R, U / C)$ are independent of $\alpha$ and $\beta$, according to Theorem 18, Theorem 28 obviously holds.

In the next section, we will present the advantages of improved fuzziness-based uncertainty on evaluating the attribute significance for a fuzzy-target information system.

\section{An Application to Fuzzy-Target Information System}

The attribute significance is an important factor in attribute reduction. The significance of an attribute is obtained by the change value of uncertainty when the attribute is removed from the whole attribute set. The larger the change is, the more the significance of the attribute is.

Definition 29. Given an information system $S=(U, C \cup$ $D, V, f), r \in C$, and $X$ is a fuzzy set on $U$, the attribute significance of $r$ can be defined as

$$
\operatorname{Sig}_{H}(r, C, D)=H_{C-\{r\}}(X)-H_{C}(X) .
$$

Definition 30. Given an information system $S=(U, C \cup$ $D, V, f), r \in C$, and $X$ is a fuzzy set on $U$, the attribute significance of $r$ can be defined as

$$
\operatorname{Sig}_{C H}(r, C, D)=C H_{C-\{r\}}(X)-C_{C}(X) \text {. }
$$

Definition 31. Given an information system $S=(U, C \cup$ $D, V, f), r \in C$, and $X$ is a fuzzy set on $U$, the attribute significance of $r$ can be defined as

$$
\operatorname{Sig}_{U N C}(r, C, D)=U N C_{C-\{r\}}(X)-U N C_{C}(X) .
$$

In this section, we will present an example to illustrate the application of the modified fuzziness-based uncertainty to a fuzzy-target information table shown in Table 1 which is artificial to address issues.

Example 32. From Table 1, we have $U=\left\{x_{1}, x_{2}, x_{3}, x_{4}, x_{5}\right.$, $\left.x_{6}, x_{7}, x_{8}, x_{9}, x_{10}\right\}$ and $X=0.1 / x_{1}+0.2 / x_{2}+0.5 / x_{3}+0.5 / x_{4}+$ $0.5 / x_{5}+0.6 / x_{6}+0.7 / x_{7}+0.8 / x_{8}+0.9 / x_{9}+0.2 / x_{10}$. Suppose
TABLE 1: A fuzzy-target information table.

\begin{tabular}{lllll}
\hline Object & $c_{1}$ & $c_{2}$ & $c_{3}$ & $D$ \\
\hline$x_{1}$ & 2 & 1 & 0 & 0.1 \\
$x_{2}$ & 0 & 1 & 2 & 0.2 \\
$x_{3}$ & 1 & 0 & 0 & 0.5 \\
$x_{4}$ & 1 & 0 & 1 & 0.5 \\
$x_{5}$ & 0 & 1 & 0 & 0.5 \\
$x_{6}$ & 0 & 1 & 2 & 0.6 \\
$x_{7}$ & 0 & 1 & 2 & 0.7 \\
$x_{8}$ & 2 & 1 & 0 & 0.8 \\
$x_{9}$ & 2 & 1 & 0 & 0.9 \\
$x_{10}$ & 2 & 1 & 0 & 0.2 \\
\hline
\end{tabular}

that $C=c_{1} \cup c_{2} \cup c_{3}, R_{1}=C-\left\{c_{1}\right\}, R_{2}=C-\left\{c_{2}\right\}, R_{3}=C-\left\{c_{3}\right\}$, then

$$
\begin{aligned}
& \left.\begin{array}{l}
U / C \\
\quad=
\end{array}\left\{x_{3}\right\},\left\{x_{4}\right\},\left\{x_{5}\right\},\left\{x_{2}, x_{6}, x_{7}\right\},\left\{x_{1}, x_{8}, x_{9}, x_{10}\right\}\right\}, \\
& \begin{array}{l}
U / R_{1} \\
\quad=
\end{array}\left\{\left\{x_{3}\right\},\left\{x_{4}\right\},\left\{x_{2}, x_{6}, x_{7}\right\},\left\{x_{1}, x_{5}, x_{8}, x_{9}, x_{10}\right\}\right\}, \\
& U / R_{2} \\
& \quad=\left\{\left\{x_{3}, x_{5}\right\},\left\{x_{4}\right\},\left\{x_{2}, x_{6}, x_{7}\right\},\left\{x_{1}, x_{8}, x_{9}, x_{10}\right\}\right\}, \\
& U / R_{3}=\left\{\left\{x_{3}, x_{4}\right\},\left\{x_{2}, x_{5}, x_{6}, x_{7}\right\},\left\{x_{1}, x_{8}, x_{9}, x_{10}\right\}\right\}
\end{aligned}
$$

According to formula (8), we have

$$
H_{C}(X)=H_{R_{1}}(X)=H_{R_{2}}(X)=H_{R_{3}}(X)=1,
$$

Therefore, we have

$$
\begin{aligned}
\operatorname{Sig}_{H}\left(c_{1}, C, D\right) & =\operatorname{Sig}_{H}\left(c_{2}, C, D\right)=\operatorname{Sig}_{H}\left(c_{3}, C, D\right) \\
& =0 .
\end{aligned}
$$

According to formula (13), we obtain

$$
\begin{aligned}
& \mathrm{CH}_{R_{1}}(X)=\mathrm{CH}_{R_{3}}(X)=0.36, \\
& \mathrm{CH}_{R_{2}}(X)=0.3, \\
& \mathrm{CH}_{C}(X)=0.28 .
\end{aligned}
$$

Therefore,

$$
\begin{aligned}
& \operatorname{Sig}_{C H}\left(c_{1}, C, D\right)=\operatorname{Sig}_{C H}\left(c_{3}, C, D\right)=0.08, \\
& \operatorname{Sig}_{C H}\left(c_{2}, C, D\right)=0.02 .
\end{aligned}
$$

According to formula (22), we compute

$$
\begin{aligned}
& U N C_{R_{1}}(X)=0.243, \\
& U N C_{R_{2}}(X)=0.15, \\
& U N C_{R_{3}}(X)=0.227, \\
& U N C_{C}(X)=0.14 .
\end{aligned}
$$


TABLE 2: Comparison between different uncertainty measure and attribute significance.

\begin{tabular}{ccccccc}
\hline & $H_{R}$ & $C_{R}$ & $U N C_{R}$ & Sig $_{H}$ & Sig $_{C H}$ & Sig $_{\mathrm{UNC}}$ \\
\hline$R_{1}$ & 1 & 0.36 & $\mathbf{0 . 2 4 3}$ & 0 & 0.08 & $\mathbf{0 . 1 0 3}$ \\
$R_{2}$ & 1 & $\mathbf{0 . 3}$ & $\mathbf{0 . 1 5}$ & 0 & $\mathbf{0 . 0 2}$ & $\mathbf{0 . 0 1}$ \\
$R_{3}$ & 1 & 0.36 & $\mathbf{0 . 2 2 7}$ & 0 & 0.08 & $\mathbf{0 . 0 8 7}$ \\
\hline
\end{tabular}

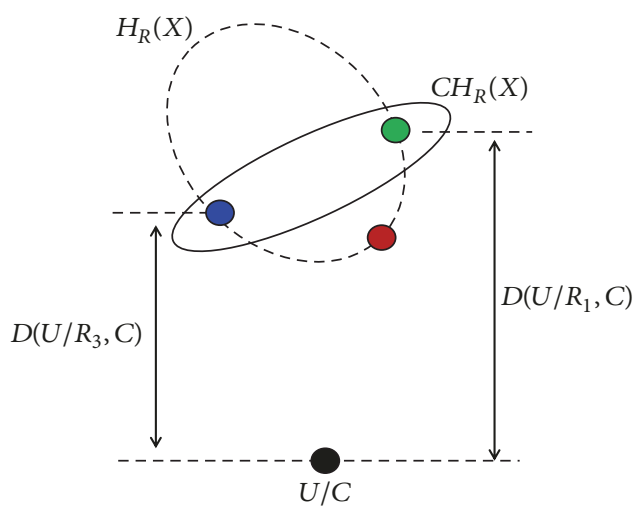

FIGURE 1: Explanation of modified uncertainty measure.

Therefore, we have

$$
\begin{aligned}
& \operatorname{Sig}_{U N C}\left(c_{1}, C, D\right)=0.103, \\
& \operatorname{Sig}_{U N C}\left(c_{2}, C, D\right)=0.01, \\
& \operatorname{Sig}_{U N C}\left(c_{3}, C, D\right)=0.087 .
\end{aligned}
$$

For convenience, the above results are listed in Table 2. From Table 2, we can obtain the following conclusions: (i) the three rough approximation spaces $U / R_{1}, U / R_{2}, U / R_{3}$ cannot be discriminated by $H_{R}$; (ii) the $\mathrm{CH}_{R_{2}}(X)$ is different from $\mathrm{CH}_{R_{1}}(\mathrm{X})$ and $\mathrm{CH}_{R_{3}}(\mathrm{X})$; (iii) although $\mathrm{CH}_{R_{1}}(\mathrm{X})=\mathrm{CH}_{R_{3}}(\mathrm{X})$, $U N C_{R}$ can distinguish $U / R_{1}$ and $U / R_{3}$. Furthermore, the attribute significance of $R_{1}, R_{2}, R_{3}$ based on the above uncertainty measures is listed in Table 2.

The above conclusions can be further described intuitively by Figure 1 . The dashed ellipse denotes the basic fuzziness-based uncertainty measure $H_{R}(X)$. The solid ellipse denotes the combined fuzziness-based uncertainty measure $\mathrm{CH}_{R}(X)$. The red, green, blue, and black dots denote the rough approximation spaces $U / R_{1}, U / R_{2}, U / R_{3}$, and $U / C$, respectively. Herein, $U / R_{1}, U / R_{2}$, and $U / R_{3}$ appear on the dashed ellipse, because $H_{R_{1}}(X)=H_{R_{2}}(X)=H_{R_{3}}(X)$; however, only $U / R_{1}$ and $U / R_{3}$ appear in the solid ellipse, because $\mathrm{CH}_{R_{2}}(\mathrm{X}) \neq \mathrm{CH}_{R_{1}}(\mathrm{X})=\mathrm{CH}_{R_{3}}(\mathrm{X})$. Furthermore, $U / R_{1}$ and $U / R_{3}$ will be distinguished as $D\left(U / R_{1}, C\right) \neq$ $D\left(U / R_{3}, C\right)$, leading to $U N C_{R_{1}}(X) \neq U N C_{R_{3}}(X)$.

Hence, according to the above results, if we take $\mathrm{CH}_{R}(X)$ to compute the attribute significance, the significance of $c_{1}$ and $c_{3}$ is equal, because $C_{R_{1}}(X)=C H_{R_{3}}(X)$. If we take $U_{N C_{R}}(X)$ to compute the attribute significance, the significance of $c_{1}$ and $c_{3}$ can be distinguished. Therefore, the deficiency mentioned in Section 4 of the traditional uncertainty measure of rough fuzzy sets is overcome effectively.

\section{Conclusions}

Rough fuzzy sets are able to deal with uncertain concepts, and the uncertainty of rough fuzzy sets usually comes from three regions. Related research on the uncertainty of rough sets and granular computing has been presented in our previous papers [46-48]. Based on these researches, in this paper, a basic fuzziness-based uncertainty measure is introduced and the essence of the fuzziness for rough fuzzy sets and its three regions in a hierarchical granular structure is revealed. From the perspective of fuzzy distance, we present a modified fuzziness-based uncertainty measure for the rough fuzzy sets. The modified measure can not only measure the information caused by the uncertainty related to the granularity of partitions, but also provide the information to distinguish the two approximation spaces with the same uncertainty. A case study is adopted to show that our method is more suitable for evaluating the significance of attributes. These results can also be applied to the uncertainty measure of probabilistic rough sets. They will have a wide variety of applications, including measuring knowledge content, constructing decision trees and building a heuristic function in a heuristic reduct algorithm in rough set theory.

\section{Data Availability}

The data used to support the findings of this study are included within the article.

\section{Conflicts of Interest}

The authors declare that they have no conflicts of interest.

\section{Acknowledgments}

This work is supported by the National Science Foundation of China (61472056), High-End Talent Project (no. RC2016005), Qian Education Cooperation (LH (2017) no. 7075), and the Key Disciplines of Guizhou Province (QXWB[2013]18).

\section{References}

[1] Z. Pawlak, "Rough sets," International Journal of Computer \& Information Science, vol. 11, no. 5, pp. 341-356, 1982.

[2] Z. Pawlak, "Rough classification," International Journal of ManMachine Studies, vol. 20, no. 5, pp. 469-483, 1984.

[3] W. Pedrycz, R. Al-Hmouz, A. Morfeq, and A. Balamash, "The design of free structure granular mappings: The use of the principle of justifiable granularity," IEEE Transactions on Cybernetics, vol. 43, no. 6, pp. 2105-2113, 2013.

[4] A. Skowron, J. Stepaniuk, and R. Swiniarski, "Modeling rough granular computing based on approximation spaces," Information Sciences, vol. 184, no. 1, pp. 20-43, 2012.

[5] Y. Y. Yao, "Information granulation and rough set approximation," International Journal of Intelligent Systems, vol. 16, no. 1, pp. 87-104, 2001.

[6] D. Dubois and H. Prade, "Twofold fuzzy sets and rough sets-some issues in knowledge representation," Fuzzy Sets and Systems, vol. 23, no. 1, pp. 3-18, 1987. 
[7] D. Dubois and H. Prade, "Rough fuzzy sets and fuzzy rough sets," International Journal of General Systems, vol. 17, no. 2-3, pp. 191-209, 1990.

[8] D. Dubois and H. Prade, Putting Rough Sets and Fuzzy Sets Together, Springer, Netherlands, 1992.

[9] B. Sun, W. Ma, and H. Zhao, "Decision-theoretic rough fuzzy set model and application," Information Sciences, vol. 283, pp. 180-196, 2014.

[10] B. Zhang and L. Zhang, Theory and applications of problem solving, Elsevier Science Inc, 1992.

[11] B. Zhang and L. Zhang, "The quotient space theory of problem solving," in International Conference on Rough Sets, Fuzzy Sets, Data Mining, and Granular Computing, vol. 2639 of Lecture Notes in Computer Science, pp. 11-15, Springer, Berlin, Germany, 2003.

[12] W. Pedrycz and W. Homenda, "Building the fundamentals of granular computing: a principle of justifiable granularity," Applied Soft Computing Journal, vol. 13, no. 10, pp. 4209-4218, 2013.

[13] W. Pedrycz, A. Skowron, and V. Kreinovich, Handbook of Granular Computing, Wiley-Interscience, 2008.

[14] G. Wang, J. Yang, and J. Xu, "Granular computing: from granularity optimization to multi-granularity joint problem solving," Granular Computing, vol. 2, no. 3, pp. 105-120, 2017.

[15] J. T. Yao, A. V. Vasilakos, and W. Pedrycz, "Granular computing: perspectives and challenges," IEEE Transactions on Cybernetics, vol. 43, no. 6, pp. 1977-1989, 2013.

[16] Y. Yao, "Perspectives of granular computing," in Proceedings of the 2005 IEEE International Conference on Granular Computing, pp. 85-90, Beijing, China, 2005.

[17] Y. Yao, "Structured Writing with Granular Computing Strategies," in Proceedings of the 2007 IEEE International Conference on Granular Computing (GRC 2007), pp. 72-72, Fremont, Calif, USA, November 2007.

[18] D. Y. Li and Y. Du, The Uncertainty Artificial Intelligence, National Defense Industry Press, 2014.

[19] L. A. Zadeh, "Fuzzy sets," Information and Computation, vol. 8, pp. 338-353, 1965.

[20] D. Y. Li and H. J. Men, "Membership and membership cloud generator," Computer Research and Development, vol. 1995, no. 6, pp. 15-20, 1965.

[21] M. J. Wierman, "Measuring uncertainty in rough set theory," International Journal of General Systems, vol. 28, no. 4-5, pp. 283-297, 1999.

[22] Y. Yao and L. Zhao, "A measurement theory view on the granularity of partitions," Information Sciences, vol. 213, pp. 113, 2012.

[23] J. Liang, K. S. Chin, C. Dang, and R. C. M. Yam, "A new method for measuring uncertainty and fuzziness in rough set theory," International Journal of General Systems, vol. 31, no. 4, pp. 331342, 2002.

[24] Y. H. Qian, J. Y. Liang, W. Wei, and F. Wang, "Consistency and fuzziness in ordered decision tables," in International Conference on Rough Sets and Knowledge Technology, pp. 63-71, 2008.

[25] J. Y. Liang, Z. Z. Shi, D. Y. Li, and M. J. Wierman, "Information entropy, rough entropy and knowledge granulation in incomplete information systems," International Journal of General Systems, vol. 35, no. 6, pp. 641-654, 2006.

[26] W. Zhang and Y. Leung, "Theory of including degrees and its applications to uncertainty inferences," in Proceedings of the Soft
Computing in Intelligent Systems and Information Processing. 1996 Asian Fuzzy Systems Symposium, pp. 496-501, Kenting, Taiwan, 1996.

[27] Z. Pawlak, "Vagueness and uncertainty: a rough set perspective," Computational Intelligence, vol. 11, no. 2, pp. 227-232, 1995.

[28] Y. Qian, Y. Li, J. Liang, G. Lin, and C. Dang, "Fuzzy Granular Structure Distance," IEEE Transactions on Fuzzy Systems, vol. 23, no. 6, pp. 2245-2259, 2015.

[29] Y. Qian, J. Liang, and C. Dang, "Knowledge structure, knowledge granulation and knowledge distance in a knowledge base," International Journal of Approximate Reasoning, vol. 50, no. 1, pp. 174-188, 2009.

[30] Z. X. Guo and J. S. Mi, "An uncertainty measure in rough fuzzy sets," Fuzzy Systems and Mathematics, vol. 19, no. 4, pp. 135-140, 2005.

[31] I. Beg and T. Rashid, "An extension of soft rough fuzzy sets," The Korean Journal of Mathematics, vol. 25, no. 1, pp. 71-85, 2017.

[32] H. Wang and W. X. Zhang, "Uncertainty measures of roughfuzzy sets," Computer Engineering and Applications, vol. 2005, no. 3, pp. 31-37, 2005.

[33] H. Qin and D. Luo, "New uncertainty measure of rough fuzzy sets and entropy weight method for fuzzy-target decisionmaking tables," Journal of Applied Mathematics, vol. 2014, 2014.

[34] B. Sun and W. Ma, "Uncertainty measure for general relationbased rough fuzzy set," Kybernetes. The International Journal of Cybernetics, Systems and Management Sciences, vol. 42, no. 6, pp. 979-992, 2013.

[35] E. C. C. Tsang, B. Z. Sun, and W. M. Ma, "General relationbased variable precision rough fuzzy set," International Journal of Machine Learning and Cybernetics, vol. 8, no. 3, pp. 1-11, 2015.

[36] J. Hu, W. Pedrycz, and G. Wang, "A roughness measure of fuzzy sets from the perspective of distance," International Journal of General Systems, vol. 45, no. 3, pp. 352-367, 2016.

[37] E. Straszecka, "Combining uncertainty and imprecision in models of medical diagnosis," Information Sciences, vol. 176, no. 20, pp. 3026-3059, 2006.

[38] R. R. Yager, "Decision making under measure-based granular uncertainty," Granular Computing, pp. 1-9, 2018.

[39] J. Dai, W. Wang, and Q. Xu, "An uncertainty measure for incomplete decision tables and its applications," IEEE Transactions on Cybernetics, vol. 43, no. 4, pp. 1277-1289, 2013.

[40] Q. Zhang, P. Zhang, and G. Wang, "Research on approximation set of rough set based on fuzzy similarity," Journal of Intelligent \& Fuzzy Systems: Applications in Engineering and Technology, vol. 32, no. 3, pp. 2549-2562, 2017.

[41] Q. H. Zhang, J. Wang, and G. Y. Wang, "Approximate representation of rough-fuzzy sets," Chinese Journal of Computers. Jisuanji Xuebao, vol. 38, no. 7, pp. 1484-1496, 2015.

[42] J. Liang, J. Wang, and Y. Qian, "A new measure of uncertainty based on knowledge granulation for rough sets," Information Sciences, vol. 179, no. 4, pp. 458-470, 2009.

[43] K. Chakrabarty, R. Biswas, and S. Nanda, "Fuzziness in rough sets," Fuzzy Sets and Systems, vol. 110, no. 2, pp. 247-251, 2000.

[44] X. C. Liu, "Entropy, distance measure and similarity measure of fuzzy sets and their relations," Fuzzy Sets and Systems, vol. 52, no. 3, pp. 305-318, 1992.

[45] Y. Qian and J. Liang, "Combination entropy and combination granulation in rough set theory," International Journal of Uncertainty, Fuzziness and Knowledge-Based Systems, vol. 16, no. 2, pp. 179-193, 2008. 
[46] Q. Zhang, Q. Zhang, and G. Wang, “The uncertainty of probabilistic rough sets in multi-granulation spaces," International Journal of Approximate Reasoning, vol. 77, pp. 38-54, 2016.

[47] Q. Zhang, S. Yang, and G. Wang, "Measuring uncertainty of probabilistic rough set model from its three regions," IEEE Transactions on Systems, Man, and Cybernetics: Systems, vol. 47, no. 12, pp. 3299-3309, 2017.

[48] J. Yang, G. Wang, and Q. Zhang, "Knowledge distance measure in multigranulation spaces of fuzzy equivalence relations," Information Sciences, vol. 448/449, pp. 18-35, 2018. 


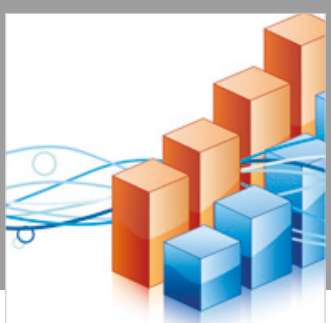

Advances in

Operations Research

\section{-n-m}
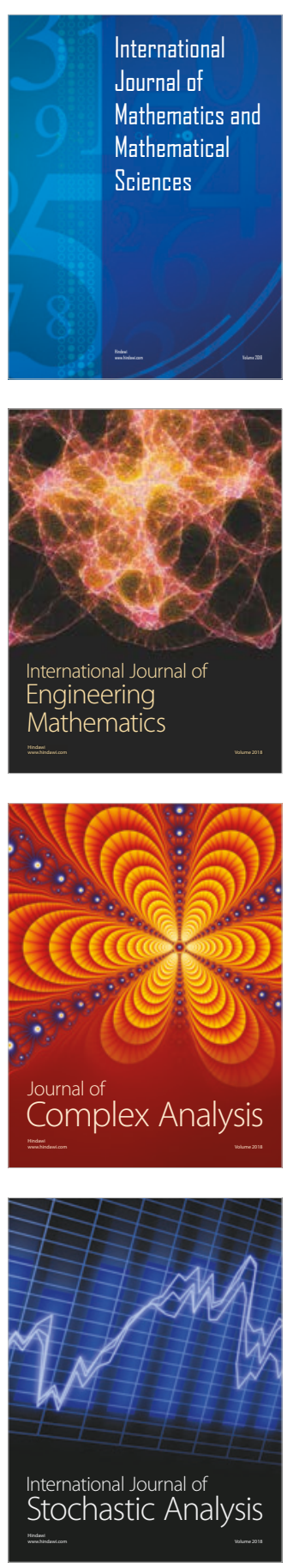
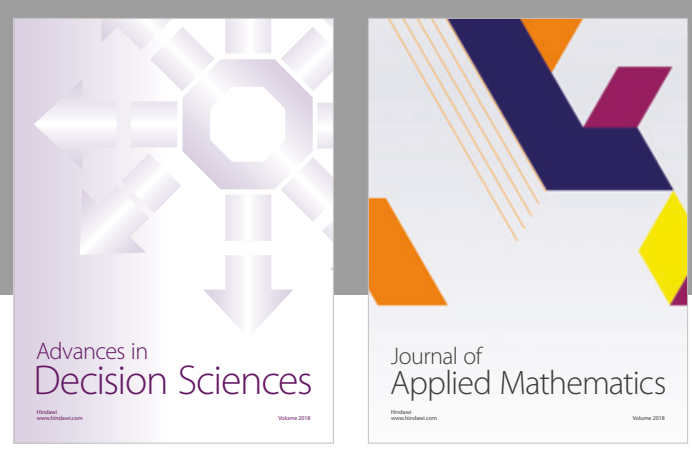

Journal of

Applied Mathematics
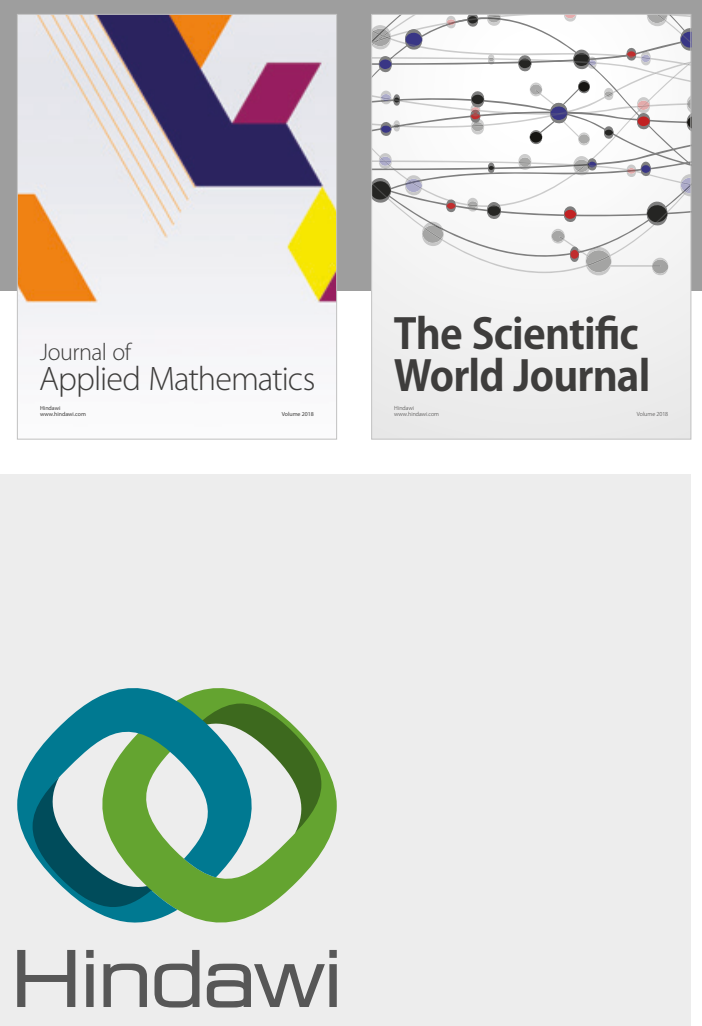

Submit your manuscripts at

www.hindawi.com

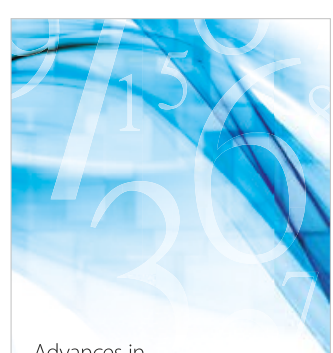

Advances in
Numerical Analysis
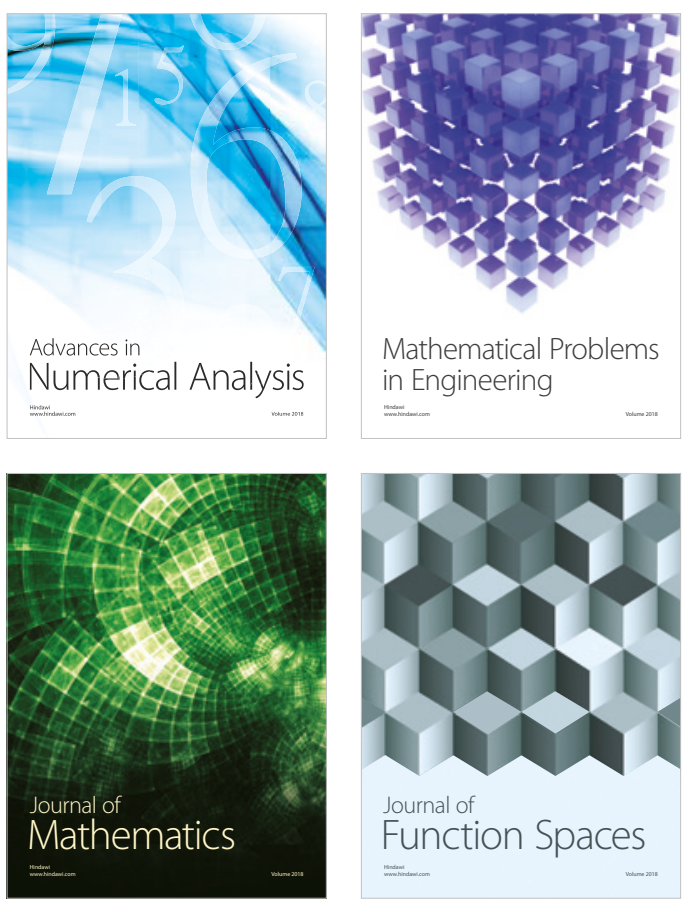

Mathematical Problems in Engineering

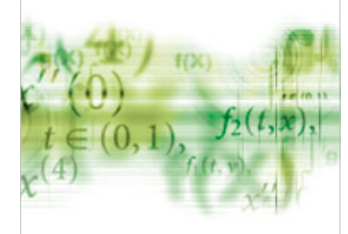

International Journal of

Differential Equations

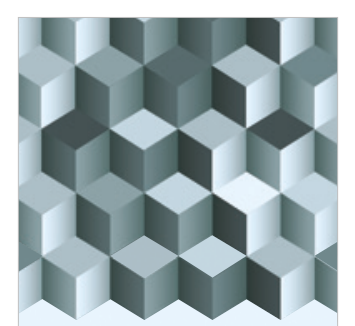

Journal of

Function Spaces

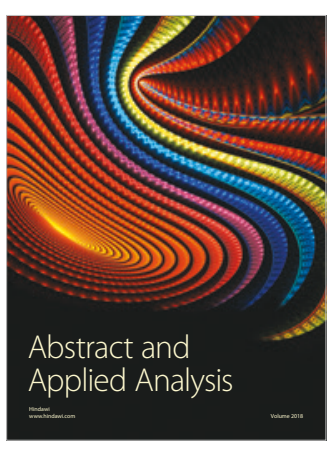

The Scientific

World Journal

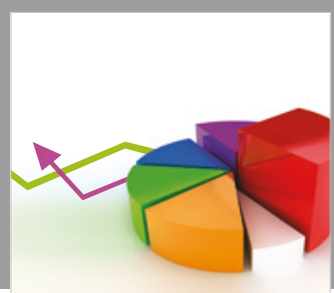

Journal of

Probability and Statistics
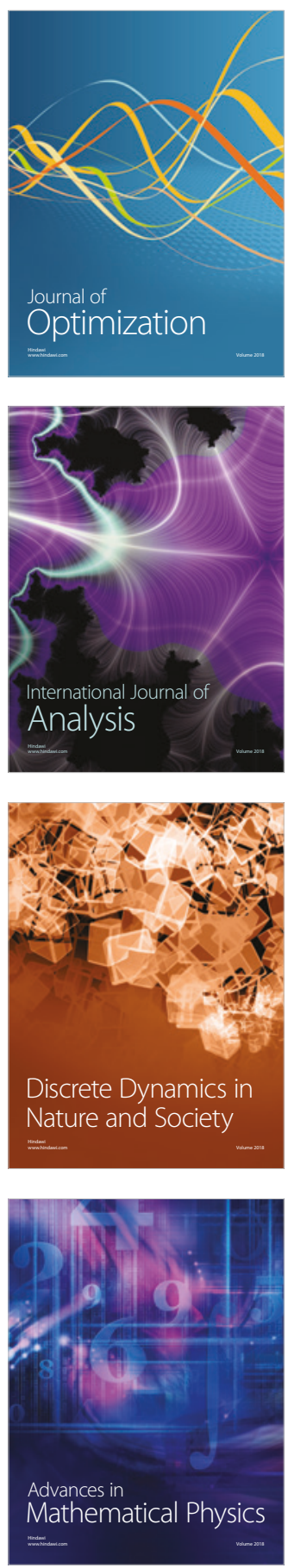